\title{
IMPORTANCIA ESTRATÉGICA DE LAS UNIDADES DE POSTGRADO EN LAS UNIVERSIDADES PÚBLICAS
}

\author{
STRATEGIC IMPORTANCE OF POSTGRADUATE UNITS OF PUBLIC \\ UNIVERSITIES IN PERU
}

\author{
Raúl Arrarte Mera ${ }^{1}$ \\ Docente Asociado de la Facultad de Ciencias Contables, UNMSM
}

(Recepción: Junio de 2009 / Conformidad: Setiembre de 2009)

\section{RESUMEN}

Las Unidades de Post Grado, en la Universidad Pública Peruana, cumplen delicadas responsabilidades estratégicas: es una fundamental fuente de ingresos propios para complementar el financiamiento de los Estudios de Pre Grado, forma Investigadores Universitarios, actualiza a los Ejecutivos tanto del Sector Público como Privado, para ser más competitivos en sus organizaciones o acrecentar su posición en el mercado laboral cada vez más exigente. Pero simultáneamente contribuye a mejorar nuestra Imagen Institucional coadyuvando al desarrollo económico y social del país.

Este artículo es un avance del trabajo de investigación en actual desarrollo realizado por el suscrito y financiado íntegramente por el Vicerrectorado de Investigaciones de la UNMSM.

Palabras clave: Postgrado, Unidades de Postgrado, Sección Postgrado.

\begin{abstract}
Units Post Grado, at the Public University Peruvian, comply sensitive strategic responsibilities is a fundamental source of own revenue to supplement the funding of the Pre-Degree Studies, is academics, updates to executives of both public and private sectors to be more competitive in their organizations and enhance their position in the labor market increasingly demanding. But at the same time it helps to improve our Institutional Image contributing to economic and social development of the country.

This article is a progress of research work under development by the undersigned and fully funded by the Vice President for Research at the UNMSM.
\end{abstract}

Key words: Graduate, Postgraduate Units, Section Postgraduate.

1 Contador Público Colegiado, Doctor en Ciencia Contables y Empresariales y Magister en Administración. Actual Director del Instituto de Investigaciones en Ciencias Financieras y Contables. E-mail: raularrarte@speedy.com.pe 


\section{INTRODUCCIÒN}

Como es por todos conocido, la Universidad Peruana se rige, desde 1984 a la fecha, por la Ley No. 23733. Y en San Marcos, su Estatuto vigente rige desde septiembre de 1984. Dichos instrumentos se hicieron en otro tiempo y en otro espacio, antes de la caída del Muro de Berlín (Noviembre de 1989), sin imaginar la actual competencia global que viene remeciendo a las organizaciones, sean éstas lucrativas o de servicio social, como es el caso de nuestra Universidad, y además, en tiempos de acreditación internacional.

Las tecnologías de información han roto el monopolio que ejercían las Universidades sobre el conocimiento. Lo que en su tiempo ocurrió con el descubrimiento de la Imprenta por Gutenberg frente al poder de la Iglesia, o el nacimiento de la Universidad Pública con la llegada de la Revolución Francesa. El usuario ahora asume su rol de «cliente» y desplaza al «alumno» que exige bienes y servicios de calidad, como es la educación que satisfaga sus necesidades inmediatas. Y si deseamos incrementar los recursos propios de la Universidad Pública, para mejorar la remuneración de nuestros Docentes, porque el Estado no aumenta su Presupuesto, caemos en la conclusión que ella sólo tiene una alterativa: adaptarse al mercado o morir en el intento. Lo que ubica a estas organizaciones, tanto públicas y privadas, en una posición de abierta competencia para captar alumnos, situación que obliga a un cambio de estrategia, y con ello a una estructura que siga a esa estrategia.

\section{EN BUSCA DE LA COMPETITIVIDAD PERDIDA.}

Según el Reglamento Interno de nuestra Universidad, los estudios de Postgrado están orientados, por un lado, al perfeccionamiento profesional a través de la Segunda Especialidad y, por otro lado, a la formación de Docentes Universitarios e Investigadores a través de la Maestría y el Doctorado. (Artículo No. 306). Sin embargo, la publicidad dirigida a la Comunidad Nacional, focaliza el concepto de «formación de docentes universitarios e investigadores». La realidad nos demuestra, que es el grueso sector de profesionales y ejecutivos del Sector Público, los que acuden a nuestras aulas universitarias en busca de actualización para ser más competitivos en su organización o mejorar su posición en el mercado laboral abierto. Como los objetivos que persiguen estos postulantes son diferentes a los de la Universidad, esta debe flexibilizar el Reglamento sobre Otorgamiento de Grados, diferenciando los procedimientos según interés del postulante.

Por ejemplo, el caso de los Docentes Universitarios procedentes de la misma Universidad, que actualmente tienen beca integral, deberían desarrollar una línea distinta de investigación. Mientras que los profesionales y ejecutivos de empresas, deberían orientarse exclusivamente a la aplicación de un proyecto específico, integrando la experiencia y los conocimientos adquiridos a través de la Mención correspondiente. Diferenciación que es fundamental explicar en los Prospectos de Admisión para captar un mayor volumen de la demanda que es cada vez más creciente y más exigente. No podemos ni debemos ir contra la corriente.

Hoy, en un ambiente de globalización, la competencia no son las universidades nacionales o públicas; son las Maestrías Internacionales con oferta completa de financiamiento: «estudie ahora y pague después» con tasas efectivas de interés preferenciales, y además, trabajo asegurado. Aunque eso ahora está por comprobarse. Los estudios son a tiempo completos durante 12 meses, y los egresados concluyen con su Grado correspondiente. Es decir, se gradúa el 100\% de la clase. ¿No podemos lograrlo en San Marcos?. He allí un objetivo al que debemos apuntar todos los que tenemos responsabilidad directa o indirecta con las Unidades de Postgrado.

Un ejemplo digno de imitar es el caso de la Escuela de Negocios de Valparaíso «Universidad Adolfo Ibáñez»(2), considerada como No. 4 en América Latina, donde se procede de la siguiente manera para optar el Grado de Magister en Administración de Empresas:

1. La Escuela de Negocios propone una lista de empresas y temas a desarrollar. Sin embargo, los estudiantes también pueden postular proyectos de su interés si cuentan con la aprobación de la empresa interesada. 
2. O a través de la participación de los estudiantes en temas específicos y concursos, tales como el organizado por el WORLD RESOURCES INSTITUTE de Washington al cual se solicita un Plan de Negocios de Empresas sostenibles.

La Tesis final es un Proyecto de Consultoría que se desarrolla en el Segundo Semestre del Programa. Finalizado el estudio los graduandos deben presentar un trabajo ante un Jurado integrado por cuatro personas: tres miembros de la Escuela y un Directivo de la empresa consultada; mientras que los primeros califican con el 50\% de la nota, el Ejecutivo Empresarial el otro 50\%. En cuanto al Profesor Asesor, se le excluye de ser parte del Jurado, asiste a la reunión de presentación con la Empresa, y luego va asesorando al Grupo de Trabajo en función al avance de la Tesis.

Para optimizar el apoyo de la empresa privada, la Escuela cuenta con un Consejo Consultivo integrado por importantes Empresarios, de suerte que proponen temas a la Universidad para que ella los tome directamente y así ahorrarse el diferencial de costos que implica pagar a una Consultora Nacional y/o Extranjera para el análisis de un tema, ya que tienen que hacer un abono de dinero a la Universidad y que sirve para financiar algunos gastos del grupo de trabajo en el desarrollo de la Tesis. El Proyecto consiste en un trabajo aplicado, real, que responde a un requerimiento concreto de una empresa o «cliente». Los alumnos trabajan directamente con la Empresa solicitante, entrevistando a ejecutivos y recolectando la información necesaria para llevar a cabo el Proyecto. Por tanto, los temas pueden ser individuales o por equipos, máximo de cinco alumnos, y siempre supervisados por un Asesor nombrado por la Escuela. Así ganan todos: la Universidad, la Empresa, el país.

\section{C Ó M O M E J O R A R L A PRODUCTIVIDAD}

Una gran debilidad de San Marcos, es su estructura organizacional. Tiene 20 Facultades y cada Facultad cuenta con una Unidad de Postgrado. La suma de las Unidades de Postgrado forma la Escuela de Postgrado. Según el Artículo 35 de su Reglamento vigente veamos cómo se maneja este organismo: «La Escuela de PostGrado de la Universidad Nacional Mayor de San Marcos depende del Rectorado y se encarga de establecer las políticas de altos estudios y de planificar, normar supervisar y coordinar las Maestrías y Doctorados».

«Cuenta con un Comité Directivo, integrado por los Directores de las Unidades de Post-Grado de las Facultades, que constituyen dos tercios, y por un tercio de estudiantes de las Maestrías y Doctorados».

El Director de la Escuela de Post-Grado, es un Profesor Principal a dedicación exclusiva o tiempo completo, con grado de Doctor, que será elegido por el Comité Directivo de la Escuela, por un período de tres (3) años. El Reglamento respectivo normará la estructura y funciones de la Escuela». En la práctica la Escuela de Postgrado sólo norma, supervisa y coordina, los Exámenes de Admisión, inicio y fin de actividades. Pero lo más importante de la organización, la planificación, se ha dejado de lado completamente, y es en ese ámbito, donde se debe trabajar en el futuro.

\section{Optimizando el Uso de Capacidad Instalada.}

Para ello hay que promover Menciones que utilicen las Aulas:

a) En las Mañanas,

b) En las Tardes,

c) Por las Noches

d) Los Fines de Semana.

Haciendo que los graduandos obtengan Maestrías y Doctorados, al término de un año, si es a tiempo completo, y a dos años si es por turno nocturno o de fines de semana.

Para cumplir con este objetivo, es necesario identificar la capacidad instalada ociosa en cada Facultad. Es decir, si cada Escuela tiene turnos de día, de tarde o de noche. Por ejemplo, ninguna Facultad, sin temor a error, tiene clases los fines de semana (sábado y domingo). Le corresponde a la Escuela de Postgrado negociar con cada Facultad, el alquiler de los salones en los fines de semana. ¿Si la Comisión de Admisión ha desarrollado cursos extraordinarios para ingresantes, por qué no puede hacer lo mismo la Escuela de Postgrado?. 
Así, la Universidad optimiza el uso de su capacidad instalada, capta mayor número de alumnos, asegura pleno empleo a sus Docentes, ahorra sus viajes a Provincias con riesgo de vida, mayor ingreso para Tesorería y beneficio para la Sociedad en su conjunto.

\section{Acelerar el Pago de Servicios a los Docentes.}

Otra debilidad que debemos superar es la celeridad en el pago de honorarios. Esta función deberá estar a cargo de la Escuela de Postgrado. Cuya meta sea pagar dentro de 7 días calendarios siguientes de cerrado el mes. Omisión que estará penado con multas a cargo de cada Facultad por incumplir con esta meta.

\section{EL CÓMO HACERLO.}

¿Qué debemos hacer para cumplir con estos objetivos?. Modificar el artículo No. 313 del Reglamento Interno, que a la letra dice, en su parte pertinente:

«Artículo 313. Los currículos de las Maestrías comprenden una duración mínima de dos años o cuatro semestres académicos».

La prioridad, para nuestra Universidad, no está en el tiempo, sino en el cumplimiento de los créditos académicos. Que se apliquen los estándares internacionales de calidad. Esto es, 80 créditos como mínimo. Por consiguiente el artículo sustitutorio que simplificaría este procedimiento sería:

«Artículo 313. Los currículos para Segunda Especialidad, Maestrías y Doctorados comprenden un mínimo de 80 créditos, los que tendrán una duración de un año o cuatro trimestres académicos si los estudios se realizan a tiempo completo. Tendrán una duración de dos años o cuatro semestres académicos, si los estudios se realizan a tiempo parcial.

Tendrán una duración de tres años o seis semestres académicos, si los estudios a tiempo parcial se realizan sólo los fines de semana (sábados y domingos)».

Asimismo, el Reglamento Interno en su Artículo No. 310, dice: «Los estudios de Maestría y Doctorado son normados, coordinados y supervisados por la Escuela de Postgrado y dirigidos y ejecutados por la Unidad de Postgrado de la Facultad por medio de sus profesores, con
Grado de Maestro o Doctor, en los ambientes respectivos......».

Como puede notarse, el Reglamento tiene una orientación unidireccional: coordinar actividades. Para ello la Escuela de Postgrado debe nombrar Cinco Comisiones:

1. Ciencias Básicas, integrada por las Unidades de Postgrado de: Biológicas, Ciencias Físicas y Ciencias Matemáticas. Formada por un Docente (Magister o Doctor), de cada Facultad.

2. Ciencias de la Salud, integrada por las Unidades de Postgrado de: Bioquímica, Farmacia, Medicina Humana, Medicina Veterinaria, Odontología y Psicología. Formada por un Docente (Magister o Doctor), de cada Facultad.

3. Ingenierías, integrada por Geología, Ingeniería de Sistemas, Ingeniería Electrónica, Ingeniería Química y Química. Formada por un Docente (Magister o Doctor), de cada Facultad.

4. Económico-Empresariales, integrada por las Unidades de Postgrado de: Ciencias Administrativas, Ciencias Contables, Ciencias Económicas y Derecho y Ciencias Políticas. Formada por un Docente (Magister o Doctor), de cada Facultad.

5. Humanidades, integrada por las Unidades de Postgrado de: Ciencias Humanas, Ciencias Sociales, Educación y Letras. Formada por un Docente (Magister o Doctor), de cada Facultad.

\section{Objetivo de cada Comisión en su Sector correspondiente:}

a. Revisar los actuales Planes de Estudios con el objeto de normalizarlos por sector para fusionar, potenciar o eliminar Menciones.

b. Normalizar el monto de las pensiones mensuales, a nivel de la Universidad.

c. Definir una política general sobre la duración de las Menciones:

- A tiempo completo, cuatro trimestres (Un año).

- A tiempo parcial, cuatro Semestres (Dos Años).

Las Frecuencias:

- Turnos de Mañanas, 
- Turnos de Tardes,

- Turnos Nocturnos.

- Turnos de Fin de Semana.

d. Identificar capacidad instalada disponible por sectores para determinar si es posible, llevar a la práctica las frecuencias propuestas en el punto anterior.

e. Conocer opinión sobre modificaciones al Reglamento Interno referido al Otorgamiento de Grados, tanto en forma individual como por equipos polivalentes (con un máximo de cinco miembros), como ya lo viene desarrollando el mercado internacional.

f. Definir procedimiento, a nivel de la Universidad, sobre la forma y manera de llevar a cabo el pago de Honorarios a favor de los Docentes del Post Grado.

\section{A MODO DE CONCLUSIÓN}

1. Facilitar la sustentación de graduandos en Maestrías y Doctorados en forma individual o por equipos polivalentes, contribuyendo a la solución de los problemas de la empresa pública y privada, es como matar dos pájaros de un solo tiro. Es actuar con los mismos instrumentos que utiliza la competencia.

2. Optimizar el uso de nuestra capacidad instalada como una sola institución, sería un gran logro no sólo para la Tesorería, sino para los Docentes. Y si conseguimos cada fin de mes abonar honorarios en su Cta. Cte. Personal, sería muestra fehaciente de haber logrado nuestra acreditación internacional.

\section{REFERENCIAS BIBLIOGRÁFICAS}

1. Ley No. 23733. Ley Orgánica Vigente de la Universidad Peruana.

2. Estatuto de la Universidad Nacional Mayor de San Marcos.

3. Portal de la Universidad Adolfo Ibáñez. Valparaíso. Chile. www.mba.uai.cl

4. Portal de la Escuela de Postgrado de UNMSM. www.unmsm.edu.pe 